\title{
Differential effects of recombinant human endostatin treatment on differentiated and undifferentiated blood vessels in Lewis lung cancer
}

\author{
WEIJIANG FU ${ }^{1}$, JING ZHUO ${ }^{2}$ and LIKUAN HU ${ }^{1}$ \\ ${ }^{1}$ Department of Radiation Oncology, Cancer Center; \\ ${ }^{2}$ Department of Ultrasonography, Qilu Hospital, Shandong University, Jinan, Shandong 250012, P.R. China
}

Received July 6, 2015; Accepted September 13, 2016

DOI: $10.3892 / \mathrm{ol} .2016 .5446$

\begin{abstract}
In the present study, we evaluated the effects of recombinant human (rh-)endostatin treatment on differentiated and undifferentiated tumor vasculature in Lewis lung cancer for the first time. Lewis lung carcinoma models were established. The animals were treated daily with varying doses of rh-endostatin or physiological saline for 14 days. Intravital microscopy was performed following treatment. The expression of CD31 and CD34 was determined by immunohistochemical staining, and microvessel density (MVD) was determined. Rh-endostatin treatment significantly decreased the tumor volume compared with the control group. Rh-endostatin treatment normalized the architecture of the vascular network. CD $31^{+}$cells decreased following rh-endostatin treatment, whereas $\mathrm{CD} 34^{+}$cells were unaffected by the treatment. Accordingly, the MVD value of $\mathrm{CD} 31^{+}$cells in rh-endostatin treatment groups significantly decreased $(\mathrm{P}<0.01)$, and the MVD value of $\mathrm{CD} 34^{+}$cells in the rh-endostatin treatment groups did not decrease. Undifferentiated tumor blood vessels were significantly inhibited by rh-endostatin treatment. In conclusion, the normalization of the tumor vasculature by endostatin may be related to the differential effects of endostatin on differentiated and undifferentiated blood vessels.
\end{abstract}

\section{Introduction}

Angiogenesis is a complex multistep biological process in which new blood vessels are formed (1). Angiogenesis is required in numerous normal physiological processes (including wound healing, embryogenesis and normal ovarian function) as well as in the pathogenesis of many disorders (including malignant

Correspondence to: Dr Likuan Hu, Department of Radiation Oncology, Cancer Center, Qilu Hospital, Shandong University, 107 Wenhua Xi Road, Jinan, Shandong 250012, P.R. China

E-mail: likuanhu1956@163.com

Key words: endostatin, Lewis lung cancer, angiogenesis, CD31, CD34 tumors) (2). Angiogenesis is regulated by a balance between pro- and anti-angiogenic molecules. However, in cancer, angiogenesis is dysregulated (2,3). In normal tissue, new blood vessels are predominantly well differentiated, while in tumors, both differentiated and undifferentiated new blood vessels are observed. In cancer patients, a poor prognosis is correlated with poor differentiation of new blood vessels $(4,5)$.

Angiogenesis is crucial for tumor growth. Therefore, creating therapies to target angiogenesis is a popular area of investigation. A number of anti-angiogenic drugs have been developed, including monoclonal antibodies and synthetic tyrosine kinase inhibitors $(6,7)$. Anti-angiogenic agents are approved for use in various types of cancer. Numerous patients have benefited from these inhibitors (8). One of these inhibitors, endostatin, has been observed to significantly suppress angiogenesis and tumor growth in a number of cancers, including Lewis lung cancer (9). However, endostatin, as well as other angiogenesis inhibitors, exhibits host toxicity, possibly due to the role angiogenesis plays in normal tissues.

Anti-angiogenic therapies are capable of improving chemotherapy efficacy by causing 'vessel normalization' in tumors (8). It has been observed that anti-angiogenic therapies transiently normalize the tumor vasculature $(10,11)$. For example, recominant human (rh-)endostatin (Endostar) normalizes the tumor vasculature and microenvironment in Lewis lung carcinoma (12). However, the mechanism of vessel normalization is unclear. We hypothesized that vessel normalization may result from the different responses of differentiated and undifferentiated tumor blood vessels to the anti-angiogenic therapies. Currently, the effects of anti-angiogenic therapies on differentiated or undifferentiated vessels have not been determined.

Tumor angiogenesis is measured by microvessel density. Two blood vessel markers, CD31 and CD34, are used to reveal different characteristics of the tumor vasculature. CD31 is expressed in all microvessels (i.e. undifferentiated and differentiated) $(13,14)$, while CD34 is highly expressed in the normal vascular endothelium, and is therefore a sensitive marker of differentiated, well-formed vessels $(15,16)$.

In the present study, we investigated the response of differentiated and undifferentiated vasculature to rh-endostatin in Lewis lung carcinoma by measuring the microvessel density. 
We also observed the normalization of tumor vessels using two-photon confocal microscopy.

\section{Materials and methods}

Cell culture and animal model. The Lewis lung carcinoma cell line was purchased from the Laboratory of Immunology, Shandong Academy of Medical Sciences, Jinan, China, and maintained in $10 \%$ fetal bovine serum.

Forty female specific pathogen-free C57BL/6 mice (Vital River Laboratories Ltd., Beijing, China) were selected for this study. The mice were 5-7 weeks of age and weighed 17-19 g. The mice were housed in groups of three and kept on a $12 \mathrm{~h}$ light/dark cycle with free access to food and water. To establish tumors, $2 \times 10^{6}$ Lewis lung carcinoma cells were subcutaneously injected into the right flank of each mouse. All experiments were approved by the Institute Animal Care and Committee of Shandong University.

Treatment protocol. Rh-endostatin was provided by Shandong Simcere-Medgenn Bio-pharmaceutical Company (Yantai, China). Once the tumors reached a length of 6-7 $\mathrm{mm}$, the mice were randomly assigned to four groups. Subcutaneous injections of rh-endostatin were administered daily at 5, 25 and $50 \mathrm{mg} / \mathrm{kg}$ for 14 days. Physiological saline was administed to the control group. Body weights were recorded daily. Tumor volumes were estimated daily using the formula $0.52 \mathrm{x}$ length $(\mathrm{mm}) \mathrm{x}$ width $\left(\mathrm{mm}^{2}\right)$, in which the length and perpendicular width were measured by calipers (12). After 14 days, the mice were sacrificed and the tumors were harvested, fixed in $10 \%$ formalin, and embedded in paraffin for subsequent experiments.

Intravital microscopy. To visualize functional blood vessels in the C57BL/6 mice, fluorescein isothiocyanate-dextran (FD2000S; Sigma-Aldrich, St. Louis, MO, USA) was injected following the 14-day rh-endostatin treatment. The dye was injected immediately prior to observation using two-photon confocal microscopy (17). The two-photon confocal microscope was comprised of an FV 300 laser confocal microscope (Olympus Corporation, Tokyo, Japan) with a 60X objective and photomultiplier tubes. A Ti:Sapphire laser source (Coherent, Inc., Santa Clara, CA, USA) with an excitation wavelength of $900 \mathrm{~nm}$ was used in the two-photon experiments. Normal skeletal muscle vessels were also observed as the normal control.

Immunohistochemistry. Four-micron serial sections were cut from the blocks of each mouse tumor. The slides were deparaffinized in xylene and rehydrated through a series of graded alcohol. High-temperature antigen retrieval was performed in a citrate salt antigen repair solution for $10 \mathrm{~min}$ in a microwave oven. After cooling to room temperature, the slides were incubated in blocking serum for $30 \mathrm{~min}$. Primary anti-CD31 antibody (1:100, rat monoclonal, ab56299; Abcam, Cambridge, MA, USA) and anti-CD34 antibody (1:50, rat monoclonal, ab8158, Abcam) were applied. The slides were incubated overnight at $4^{\circ} \mathrm{C}$ in a high humidity chamber. Certain sections were incubated in phosphate-buffered saline as a negative control. After washing, the tissue sections were treated with biotinylated goat-anti-rat secondary antibody (Zhongshan

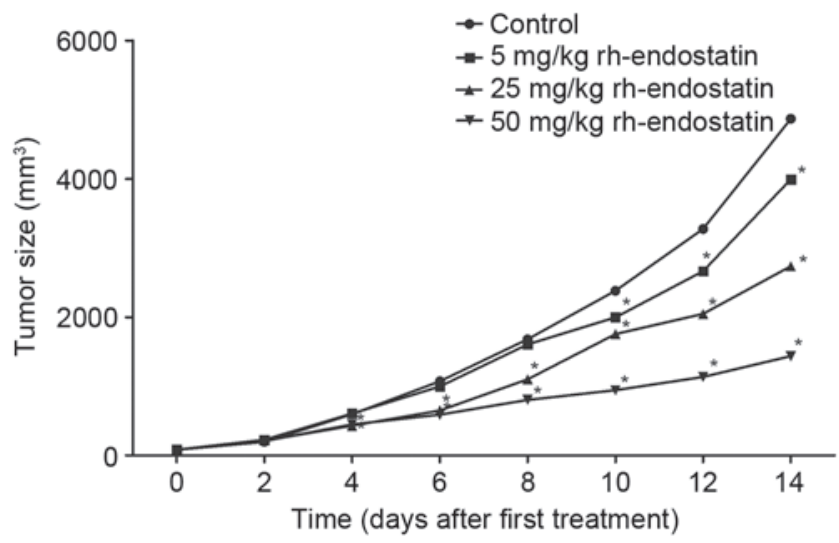

Figure 1. Recombinant human (rh-)endostatin treatment inhibits tumor formation. Animals were treated for 14 days with saline (control), $5 \mathrm{mg} / \mathrm{kg}$ rh-endostatin, $25 \mathrm{mg} / \mathrm{kg}$ rh-endostatin or $50 \mathrm{mg} / \mathrm{kg}$ rh-endostatin. Tumor volumes were significantly reduced in the rh-endostatin-treated animals compared with those in control animals $\left({ }^{*} \mathrm{P}<0.05\right)$.

Biotechnology Company, Beijing, China) and further incubated with streptavidin-horseradish peroxidase complex for $20 \mathrm{~min}$. Sections were finally stained with diaminobenzidine and counterstained with hematoxylin and eosin.

Microvessel density. Microvessel density (MVD) was determined according to the method described by Huang and Chen (12). Briefly, two researchers independently assessed MVD. Any $\mathrm{CD} 1^{+}$or $\mathrm{CD}_{3} 4^{+}$endothelial cells or cell clusters that were clearly separated from the surrounding tumor and stromal cells were counted as microvessels. The sections were screened at lower magnifications (x100) to identify five vascularized areas. Within the selected areas, microvessels were counted under high magnification (x400). The MVD was the average number of microvessels in the five fields. Discrepancies were resolved through discussion and reviewing the section.

Statistical analysis. Data were expressed as the means \pm standard error. The Statistical Package for the Social Sciences (SPSS) version 19.0 (IBM SPSS, Armonk, NY, USA) was used for statistical analysis. Statistical significance was determined by one-way analysis of variance. The least significant difference test was applied for multiple means comparisons. $\mathrm{P}<0.05$ was considered to represent a statistically significant difference.

\section{Results}

Rh-endostatin inhibits tumor growth in a dose-dependent manner. Following the 14-day treatment with rh-endostatin, tumor growth was inhibited (Fig. 1). Tumor volumes were significantly reduced in the rh-endostatin groups compared with those in the control group $(3,991.5 \pm 761.9,2,735.1 \pm 558.3$ and $1,433.9 \pm 275.3 \mathrm{~mm}^{3}$, respectively, in the 5,25 and $50 \mathrm{mg} / \mathrm{kg}$ groups vs. $4,869.8 \pm 990.0 \mathrm{~mm}^{3}$ in the control group; $\mathrm{P}<0.05$ ). Treatment with $50 \mathrm{mg} / \mathrm{kg}$ rh-endostatin was significantly more effective in tumor growth inhibition than the other two doses $(\mathrm{P}<0.05)$. This data confirms that rh-endostatin effectively inhibits tumor growth. 
A

B

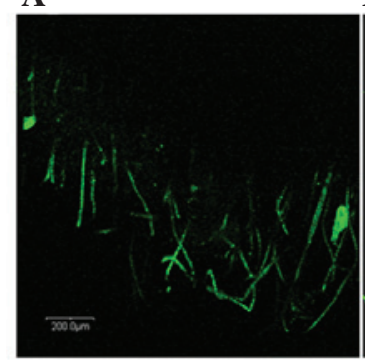

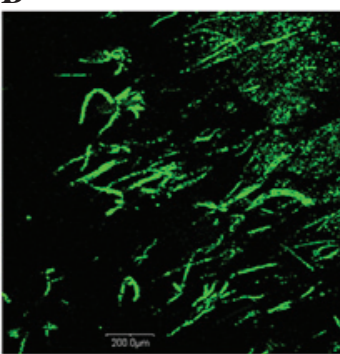

C

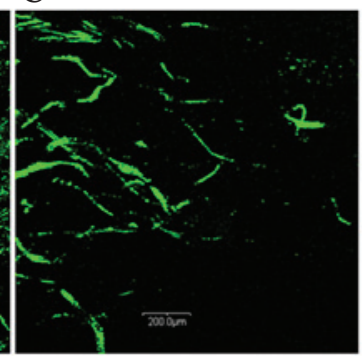

D

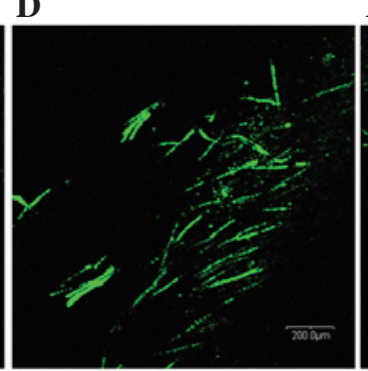

E

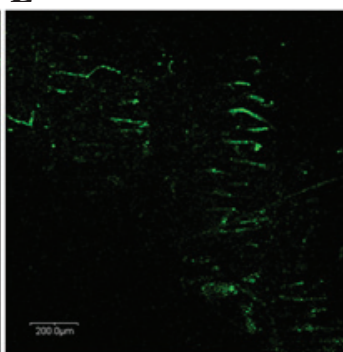

Figure 2. Tumor vasculature is normalized following recombinant human (rh-)endostatin treatment. Following the 14-day endostatin treatment, the tumor vasculature was imaged by intravital two-photon confocal microscopy. Rh-endostatin normalized the architecture of the tumor vascular network. The treatment groups were compared with images of normal vessels in the skeletal muscle (A). The animals were treated with (B) saline, (C) 5 mg/kg rh-endostatin, (D) $25 \mathrm{mg} / \mathrm{kg}$ rh-endostatin or (E) $50 \mathrm{mg} / \mathrm{kg}$ rh-endostatin. Blood vessels were contrast enhanced by fluorescein isothiocyanate-dextran, which was injected through the rat tail vein prior to visualization by two-photon confocal microscopy. Magnification, x300.

A
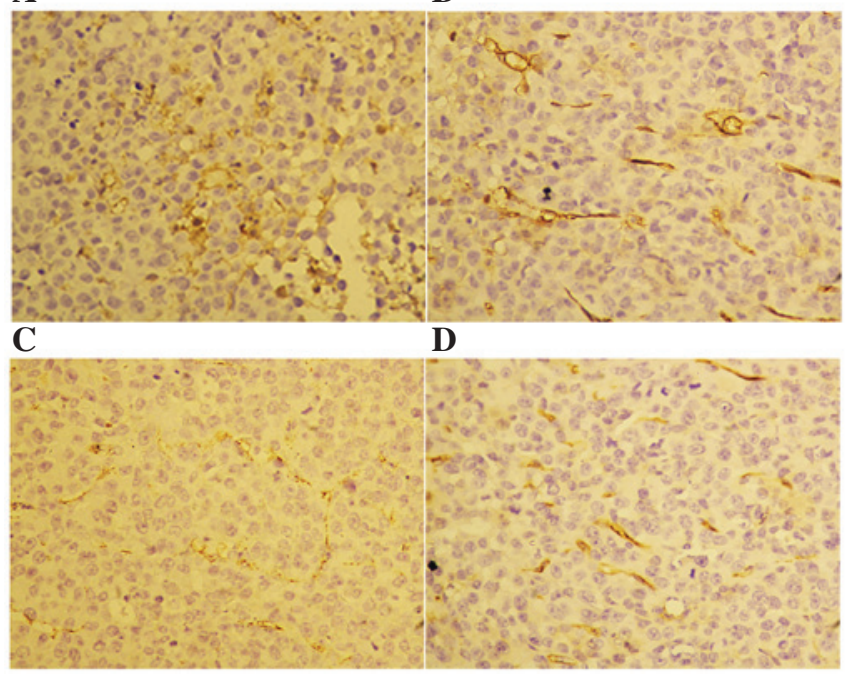

Figure 3. Immunohistochemical analysis of CD31 in Lewis lung cancer. Recombinant human (rh-)endostatin treatment decreases CD31 staining. The animals were treated with saline (A), $5 \mathrm{mg} / \mathrm{kg}$ rh-endostatin (B), $25 \mathrm{mg} / \mathrm{kg}$ rh-endostatin (C), or $50 \mathrm{mg} / \mathrm{kg}$ rh-endostatin (D). Magnification, $\mathrm{x} 400$.

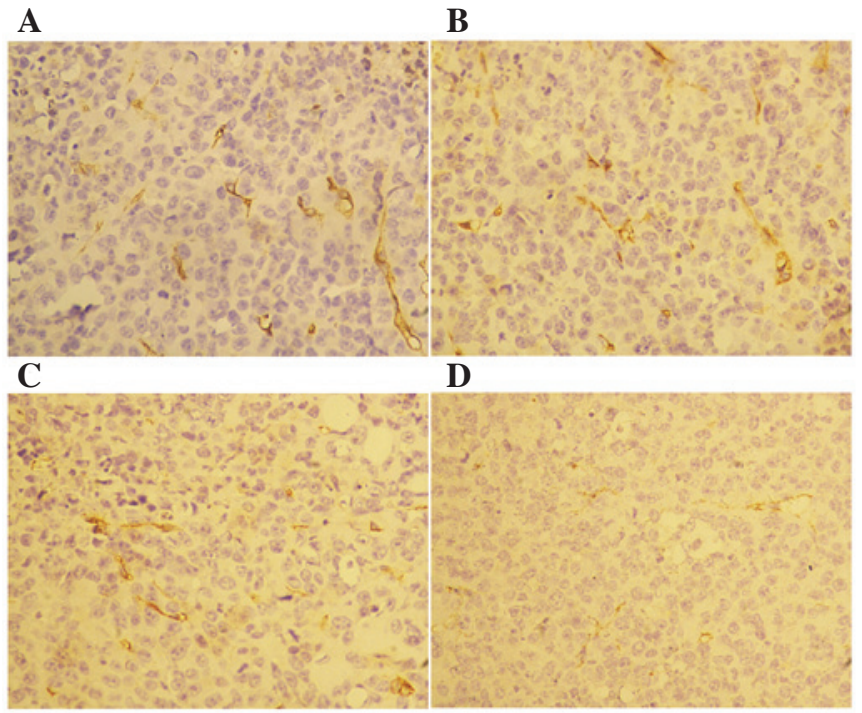

Figure 4. Immunohistochemical analysis of CD34 in Lewis lung cancer. Recombinant human (rh-)endostatin treatment does not affect CD34 staining. The animals were treated with saline (A), $5 \mathrm{mg} / \mathrm{kg}$ rh-endostatin (B), $25 \mathrm{mg} / \mathrm{kg}$ rh-endostatin (C), or $50 \mathrm{mg} / \mathrm{kg}$ rh-endostatin (D). Magnification, x400.

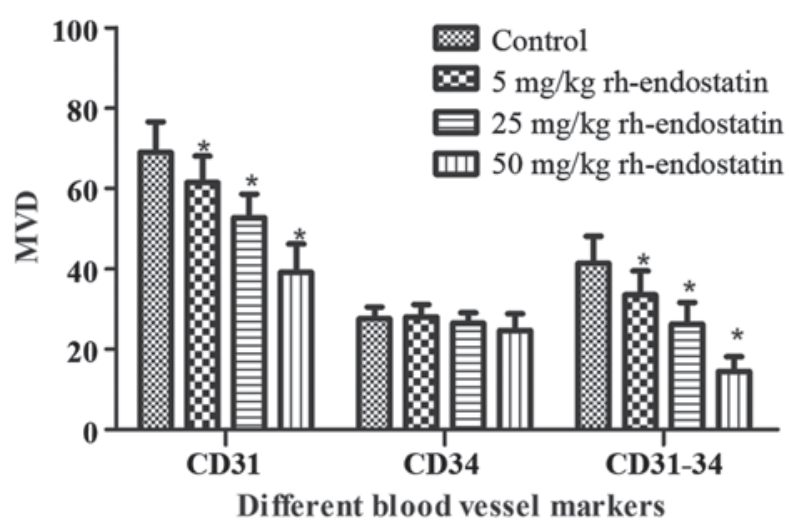

Figure 5. Microvessel density (MVD) is decreased in $\mathrm{CD} 31^{+}$cells but not in $\mathrm{CD}_{3} 4^{+}$cells following recombinant human (rh-)endostatin treatment. Animals were treated with saline (control), $5 \mathrm{mg} / \mathrm{kg}$ rh-endostatin, $25 \mathrm{mg} / \mathrm{kg}$ rh-endostatin or $50 \mathrm{mg} / \mathrm{kg}$ rh-endostatin for 14 days. MVD was determined by averaging the number of positive cells or cell clusters in five vascularized regions. The number of undifferentiated vessels was determined by subtracting the $\mathrm{CD} 34^{+}$cells from the $\mathrm{CD} 31^{+}$cells $(\mathrm{CD} 31-\mathrm{CD} 34)\left({ }^{*} \mathrm{P}<0.05\right)$.

Rh-endostatin normalizes the architecture of the tumor vasculature. We examined the effect of rh-endostatin on the morphology of blood vessels in the Lewis lung cancer tumors by confocal microscopy. Normal skeletal muscle has an organized vasculature with a relatively smooth vascular wall and uniform diameter (Fig. 2A). In contrast, the Lewis lung cancer tumor in the control group had abundant tortuous vessels with abrupt changes in vessel diameter and a number of extremely small vessels (Fig. 2B). However, in the tumors treated with rh-endostatin, the vessels became less tortuous and more regular, and assumed a relatively normal morphology as the dose increased (Fig. 2C-E). These data provide further evidence that rh-endostatin normalizes the architecture of the vascular network.

Rh-endostatin affects differentiated and undifferentiated blood vessels differently. Following the 14-day treatment with rh-endostatin, we observed a decreased number of CD31 ${ }^{+}$ cells in the tumor blood vessels (Fig. 3). However, we did not observe any differences in CD34 staining between the control and rh-endostatin-treated groups (Fig. 4). The MVD of $\mathrm{CD} 31^{+}$cells significantly decreased following treatment with rh-endostatin $(61.6 \pm 6.53,52.8 \pm 5.8$ and $39.2 \pm 6.94$, respectively, 
in the 5,25 and $50 \mathrm{mg} / \mathrm{kg}$ rh-endostatin groups vs. $69.0 \pm 7.62$ in the control group; $\mathrm{P}<0.05$ ) (Fig. 5). This decrease occurred in a dose-dependent manner. Furthermore, the MVD of CD $34^{+}$cells was similar in the treatment and control groups $(28.0 \pm 3.15,26.5 \pm 2.54$ and $24.7 \pm 4.13$, respectively, in the 5, 25 and $50 \mathrm{mg} / \mathrm{kg}$ rh-endostatin groups vs. $27.5 \pm 2.98$ in the control group; $\mathrm{P}>0.05$ ) (Fig. 5).

According to previous studies, undifferentiated blood vessels may be quantified by $\mathrm{CD} 31^{+} \mathrm{CD} 34^{-}$cells or by subtracting the $\mathrm{CD} 34^{+} \mathrm{MVD}$ value from the CD $31^{+} \mathrm{MVD}$ value $(4,18)$. We observed that the number of undifferentiated blood vessels (CD31-CD34) was significantly decreased following rh-endostatin treatment (Fig. 5). Accordingly, the decrease occurred in a dose-dependent manner $(33.5 \pm 5.97$, $26.3 \pm 5.33$ and $14.5 \pm 3.61$, respectively, in the 5, 25 and $50 \mathrm{mg} / \mathrm{kg}$ rh-endostatin groups vs. $41.44 \pm 6.62$ in the control group; $\mathrm{P}<0.01)$.

\section{Discussion}

In the present study, we evaluated the effects of recombinant human (rh-)endostatin treatment on differentiated and undifferentiated tumor vasculature in Lewis lung cancer for the first time. We detected a change in tumor microvessel density, the 'gold standard' of assessing tumor angiogenesis, following treatment with varying doses of rh-endostatin. The tumor blood vessel cells with positive CD31 staining represent both differentiated and undifferentiated endothelial cells, whereas cells with positive CD34 staining represent only differentiated endothelial cells $(4,18)$. Following rh-endostatin treatment, CD $31^{+}$cells significantly decreased in a dose-dependent manner. However, no significant reduction in differentiated neovascularization $\left(\mathrm{CD} 34^{+}\right.$cells) was observed. These data led us to conclude that rh-endostatin primarily inhibited the undifferentiated $\left(\mathrm{CD} 31^{+} \mathrm{CD} 34^{-}\right)$neovascularization. This is the first study to observe the differential effects of rh-endostatin on differentiated and undifferentiated neovascularization.

The role played by endostatin, an efficient anti-angiogenic and antitumor molecule, in angiogenesis inhibition is still unclear. It may directly affect cells undergoing angiogenesis or regulate the secretion of various growth factors $(9,19-21)$. Endostatin was first isolated from the supernatant of a murine hemangio-endothelioma, and it almost completely suppressed the formation of new blood vessels (9). Since then, numerous studies have investigated the effect of endostatin on angiogenesis (19-21) and on tumor vessel normalization $(12,22)$. However, none of these studies focused on the differential effects of rh-endostatin on differentiated and undifferentiated blood vessels. Our research suggests that rh-endostatin has a stronger effect on undifferentiated blood vessels than on differentiated blood vessels. This result provided further insight into the mechanism of tumor vessel normalization by endostatin.

In addition, we directly observed the effect of rh-endostatin on tumor vasculature in Lewis lung cancer using intravital two-photon confocal microscopy. Imaging revealed that following rh-endostatin treatment, the tumor vessels were similar to normal vessels. Tumor microvessels may be observed directly in real time and in vivo by two-photon microscopy (17). Several studies have demonstrated tumor vessel normalization by anti-angiogenic drugs using multi-photon confocal microscopy. For example, Tong et al (23) demonstrated the normalization process in four tumor types following DC101 treatment. In addition, von Baumgarten et al (24) observed the normalization of glioma blood vessels following bevacizumab treatment. However, the present study is the first to report the use of intravital confocal microscopy to visualize tumor vessel normalization in Lewis lung cancer following endostatin treatment.

In conclusion, we observed that rh-endostatin significantly inhibited the formation of undifferentiated vasculature $\left(\mathrm{CD} 31^{+} / \mathrm{CD} 34^{-}\right)$but did not inhibit the formation of differentiated vasculature $\left(\mathrm{CD} 31^{+} / \mathrm{CD} 34^{+}\right)$. Normalization of the tumor blood vessels was also observed. Taken together, these results suggest that normalization of the tumor vasculature by endostatin may be related to the differential effects of endostatin on differentiated and undifferentiated blood vessels. Further study into the mechanism of tumor vessel normalization by endostatin is required in order to apply these findings to the clinic.

\section{Acknowledgements}

This study was supported by the Shandong Province Science and Technology Development Plan [2014GGE27076]. The authors wish to thank Dr. Edward C. Mignot from Shandong University for his linguistic advice.

\section{References}

1. Gacche RN and Meshram RJ: Targeting tumor micro-environment for design and development of novel anti-angiogenic agents arresting tumor growth. Prog Biophys Mol Biol 113: 333-354, 2013.

2. Wietecha MS, Cerny WL and DiPietro LA: Mechanisms of vessel regression: toward an understanding of the resolution of angiogenesis. Curr Top Microbiol Immunol 367: 3-32, 2013.

3. Zhang Y, Yu LK, Lu GJ, Xia N, Xie HY, Hu W, Hao KK, Xu CH and Qian Q: Prognostic values of VEGF and endostatin with malignant pleural effusions in patients with lung cancer. Asian Pac J Cancer Prev 15: 8435-8440, 2014.

4. Yao X, Qian CN, Zhang ZF, Tan MH, Kort EJ, Yang XJ, Resau JH and Teh BT: Two distinct types of blood vessels in clear cell renal cell carcinoma have contrasting prognostic implications. Clin Cancer Res 13: 161-169, 2007.

5. Qi L, Du J, Zhang Z, Diao L, Chen X and Yao X: Low differentiated microvascular density and low expression of platelet-derived growth factor-BB (PDGF-BB) predict distant metastasis and poor prognosis in clear cell renal cell carcinoma. BJU Int 112: E415-E423, 2013.

6. Fontanella C, Ongaro E, Bolzonello S, Guardascione M, Fasola G and Aprile G: Clinical advances in the development of novel VEGFR2 inhibitors. Ann Transl Med 2: 123, 2014.

7. Welti J, Loges S, Dimmeler S and Carmeliet P: Recent molecular discoveries in angiogenesis and antiangiogenic therapies in cancer. J Clin Invest 123: 3190-3200, 2013.

8. Jain RK: Antiangiogenesis strategies revisited: from starving tumors to alleviating hypoxia. Cancer Cell 26: 605-622, 2014.

9. O'Reilly MS, Boehm T, Shing Y, Fukai N, Vasios G, Lane WS, Flynn E, Birkhead JR, Olsen BR and Folkman J: Endostatin: an endogenous inhibitor of angiogenesis and tumor growth. Cell 88: 277-285, 1997.

10. Jain RK: Normalization of tumor vasculature: an emerging concept in antiangiogenic therapy. Science 307: 58-62, 2005.

11. Huang Y, Yuan J, Righi E, Kamoun WS, Ancukiewicz M, Nezivar J, Santosuosso M, Martin JD, Martin MR, Vianello F, et al: Vascular normalizing doses of antiangiogenic treatment reprogram the immunosuppressive tumor microenvironment and enhance immunotherapy. Proc Natl Acad Sci USA 109: 17561-17566, 2012. 
12. Huang $\mathrm{G}$ and Chen L: Recombinant human endostatin improves anti-tumor efficacy of paclitaxel by normalizing tumor vasculature in Lewis lung carcinoma. J Cancer Res Clin Oncol 136: 1201-1211, 2010.

13. Giatromanolaki A, Koukourakis MI, Theodossiou D, Barbatis K, O'Byrne K, Harris AL and Gatter KC: Comparative evaluation of angiogenesis assessment with anti-factor-VIII and anti-CD31 immunostaining in non-small cell lung cancer. Clin Cancer Res 3: 2485-2492, 1997.

14. Caraffi S, Corradi D, Campanini N, Govoni P, Rocchi L, Perris R and Mangieri D: Microcirculation density and maturity in uterine and soft tissue leiomyosarcomas: an immunohistochemical study. Histol Histopathol 30: 69-76, 2015.

15. Suster S and Wong TY: On the discriminatory value of anti-HPCA-1 (CD-34) in the differential diagnosis of benign and malignant cutaneous vascular proliferations. Am J Dermatopathol 16: 355-363, 1994.

16. Miyata Y, Mitsunari K, Asai A, Takehara K, Mochizuki Y and Sakai H: Pathological significance and prognostic role of microvessel density, evaluated using CD31, CD34, and CD105 in prostate cancer patients after radical prostatectomy with neoadjuvant therapy. Prostate 75: 84-91, 2015.

17. Fukumura D, Duda DG, Munn LL and Jain RK: Tumor microvasculature and microenvironment: novel insights through intravital imaging in pre-clinical models. Microcirculation 17 206-225, 2010
18. Poblet E, Gonzalez-Palacios F and Jimenez FJ: Different immunoreactivity of endothelial markers in well and poorly differentiated areas of angiosarcomas. Virchows Arch 428: 217-221, 1996.

19. Barczyk M, Carracedo S and Gullberg D: Integrins. Cell 339: 269-280, 2010.

20. Dong XP, Xiao TH, Dong H, Jiang N and Zhao XG: Endostar combined with cisplatin inhibits tumor growth and lymphatic metastasis of lewis lung carcinoma xenografts in mice. Asian Pac J Cancer Prev 14: 3079-3083, 2013.

21. Xiao L, Yang S, Hao J, Yuan X, Luo W, Jiang L, Hu Y, Fu Z, Zhang Y and Zou C: Endostar attenuates melanoma tumor growth via its interruption of b-FGF mediated angiogenesis. Cancer Lett 359: 148-154, 2015.

22. Peng F, Xu Z, Wang J, Chen Y, Li Q, Zuo Y, Chen J, Hu X, Zhou Q, Wang Y, et al: Recombinant human endostatin normalizes tumor vasculature and enhances radiation response in xenografted human nasopharyngeal carcinoma models. PLoS One 7: e34646, 2012.

23. Tong RT, Boucher Y, Kozin SV, Winkler F, Hicklin DJ and Jain RK: Vascular normalization by vascular endothelial growth factor receptor 2 blockade induces a pressure gradient across the vasculature and improves drug penetration in tumors. Cancer Res 64: 3731-3736, 2004.

24. von Baumgarten L, Brucker D, Tirniceru A, Kienast Y, Grau S, Burgold S, Herms J and Winkler F: Bevacizumab has differential and dose-dependent effects on glioma blood vessels and tumor cells. Clin Cancer Res 17: 6192-6205, 2011. 